Gefässchirurgie 2009 · 14:5-5 DOI 10.1007/s00772-008-0657-x

(C) Springer Medizin Verlag 2009

\title{
Online-Einreichung von Manuskripten für die "Gefässchirurgie"
}

mehr nötig. Die Online-Einreichung bie tet die automatische Datenkonversion der Urdatei in eine PDF-Datei - ein stabiles Dokument, das überall an jedem Computer gleich aussieht.

- Beschleunigt den Begutachtungsprozess: Das Redaktionssystem ermöglicht die nahtlose Verbindung zwischen Einreichung, Begutachtung, eventueller Revision und schließlich Veröffentlichung.

- Ihre Daten sind sicher: Sie werden auf dem sicheren zentralen Datenserver
Chirurgische Klinik und Poliklinik

Zentrum operative Medizin

Abteilung für vaskuläre und

endovaskuläre Chirurgie

Oberdürrbacher Straße 6

97080 Würzburg

Larena_a@chirurgie.uni-wuerzburg.de gespeichert, der regelmäßig gesichert wird, sodass ein Datenverlust nicht vorkommt.

- Spart Ihnen Kosten: Es ist kein teurer Postversand mehr nötig. Die Einreichung ist in kürzester Zeit nach Ihrer Einspeisung und Bestätigung beim Herausgeber.

Weitere Informationen zum Verfassen und Einreichen von Manuskripten finden Sie in der nebenstehenden Infobox.

Ihr

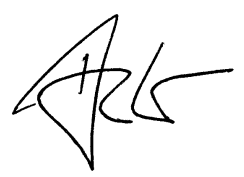

Prof. Dr. E.S. Debus

Schriftleitung "Gefässchirurgie“

\section{Schriftleitung}

Prof. Dr. E. Sebastian Debus

Abt. Allgemein-, Gefäß- und Visceralchirurgie GefäßCentrum Hamburg Harburg

Asklepios Klinik Harburg

Eißendorfer Pferdeweg 52

21075 Hamburg

s.debus@asklepios.com

\section{Infobox}

Manuskript verfassen

- Bitte folgen Sie auf der Homepage www.Gefaesschirurgie.springer.de dem Link „Für Autoren“.

- Hier finden Sie die Autorenleitfäden und Musterbeiträge mit Hinweisen für die Manuskriptgestaltung.

Manuskript einreichen

- Bitte klicken Sie auf „Manuskript online einreichen" und anschließend auf die Schaltfläche „Editorial Manager".

- Bei der ersten Nutzung registrieren Sie sich unter "Register" mit der Angabe einer Benutzer-ID und eines Passwortes. Damit haben Sie bei jeder weiteren Publikation direkten Zugang zum System.

- Nach der Anmeldung finden Sie unter "Author Login" alle notwendigen Hinweise, wie Sie Ihren Beitrag einreichen können.

Sollten Sie noch Fragen zur Manuskripteinreichung haben, wenden Sie sich bitte an:

\section{PD Dr. Axel Larena-Avellaneda}

Chirurgische Klinik und Poliklinik Zentrum operative Medizin Abteilung für vaskuläre und endovaskuläre Chirurgie Oberdürrbacher Straße 6 97080 Würzburg

Larena_a@chirurgie.uni-wuerzburg.de

\section{Assistenz der Schriftleitung}

PD Dr. Axel Larena-Avellaneda

www.Gefaesschirurgie.springer.de 